\title{
Short-term fenofibrate treatment improves ultrastructure of hepatocytes of old rats
}

\author{
Adrian Zubrzycki, Agata Wronska, Agata Zauszkiewicz-Pawlak, Zbigniew Kmiec
}

\author{
Department of Histology, Medical University of Gdansk, Gdansk
}

\begin{abstract}
Introduction. Fenofibrate (FN) is a hypolipemic drug used for the treatment of mixed dyslipidemia. Since in our previous study FN administration to young and old rats adversely affected the serum activity of liver marker enzymes, we decided to examine the effects of FN on liver ultrastructure of young and old animals.

Material and methods. Young and old rats were fed standard rodent chow supplemented with $0.1 \% \mathrm{FN}$ for 30 days. Liver samples obtained from animals under full anesthesia were processed by routine methods to obtain ultrathin and histological sections for the examination by light microscopy (LM) and transmission electron microscopy (TEM). Furthermore, liver lysates were analyzed by Western blotting for the expression of the autophagy-related proteins LC3A/B and beclin 1.

Results. The ultrastructure of hepatocytes in both age groups was well-preserved, with the presence of abundant mitochondria, numerous peroxisomes and lysosomes, glycogen stored in the form of rosettes, and occasionally autolysosomes. However, hepatocytes of old control rats contained less mitochondria and peroxisomes, and more lipid droplets than cells of young animals. The effects of FN on liver ultrastructure were age-depended. FN increased the relative number of mitochondria and peroxisomes in the hepatocytes of old, and did not affect their number in young rats. Moreover, FN decreased and increased the relative number of lipid droplets in the hepatocytes of old and young rats, respectively. At the LM level, Oil Red O staining revealed smaller and larger lipid droplets within hepatocytes and non-parenchymal liver cells. In the livers of young and old rats lipid droplets were distributed mainly in the periportal zones of hepatic lobules. Morphometric analysis confirmed that livers of control old rats contained more lipid-stainable areas than those of young ones; however, no effect of FN was observed either in young or old rats. Despite larger size of autolysosomes and autophagic vacuoles in hepatocytes of old rats, the expression of autophagy-related proteins did not differ in the livers of control and fenofibrate-treated young and old animals.

Conclusions. The results of our study suggest that fenofibrate, apart from its hypolipemic action, may have beneficial effect on the energy metabolism in the liver of old individuals by increasing the number of mitochondria and peroxisomes in hepatocytes. (Folia Histochemica et Cytobiologica 2021, Vol. 59, No. 3, 167-177)
\end{abstract}

Key words: fenofibrate; aging; rat; liver; hepatocyte; lipid droplets; LC3A/B; beclin 1; TEM

\section{Introduction}

Fenofibrate (FN) has been used to correct lipid abnormalities in patients with dyslipidemias $[1,2]$.

\footnotetext{
Correspondence address: Adrian Zubrzycki, MSc

Medical University of Gdansk,

Department of Histology,

1 Debinki Str., 80-211 Gdansk, Poland

e-mail: adrian.zubrzycki@gumed.edu.pl
}

Hypolipemic effects of FN are related to the activation of a class of intracellular receptors, known as peroxisome proliferator-activated receptors (PPARs), especially $\operatorname{PPAR} \alpha$ in the liver [3]. These receptors modulate carbohydrate and lipid metabolism by the up- or downregulation of the transcription of multiple genes that are responsible for the regulation of hepatic lipid metabolism [4, 5].

Depending on the dose, FN can induce adverse side effects in the liver [6, 7]. In our previous work we showed that, apart from beneficial effects on the 
levels of serum lipids, FN administration for 30 days to young and old rats was associated with alterations of liver histological structure and increased blood serum activity of alkaline phosphatase [8]. Other studies showed that $\operatorname{PPAR} \alpha$ activation by fibrates induces in rodents strong proliferation of peroxisomes, resulting in hyperplasia and hypertrophy of hepatocytes [9-12]. In liver-specific humanized PPAR $\alpha$ transgenic mice, FN caused proliferation of liver cells and hepatocyte hypertrophy, dependent on the dose and duration of FN treatment [12]. Moreover, PPAR $\alpha$ activation decreased apoptosis in an experimental rat cholestasis model [9]. However, the effects of $\operatorname{PPAR} \alpha$ ligands, such as FN, on hepatocyte and peroxisome proliferation differ between rodents and humans [13].

Even though biological effects of FN have been studied almost for forty years, there is scarcity of data on age-dependent effects of the drug. In our previous study performed on old and young rats we found, beside the expected hypolipemic action of FN, also some indices of adverse effects of $\mathrm{FN}$ on the liver function and structure at the light-microscopic level. Therefore, in the present study we decided to compare FN's effects on hepatocytes' ultrastructure in young and old healthy Wistar-Han rats, and on the expression of autophagy-related proteins, i.e. LC3A/B which is related to autophagosome formation, and beclin 1 , a common regulator of autophagy and apoptosis [14].

\section{Materials and methods}

Animals. The experiment was carried out on young (4-month-old) and old (24-month-old) male Wistar-Han rats, bred in the Academic Animal Experimental Center in Gdansk, Poland, and housed one per cage in standard breeding conditions $\left(22 \pm 2^{\circ} \mathrm{C}\right.$, humidity $55 \pm 10 \%, 12 \mathrm{~h} / 12 \mathrm{~h}$ light/ /dark cycle). The experimental procedures were approved by the Local Ethics Committee in Bydgoszcz, Poland (protocols No. 41/2017, 58/2017, 40/2018, and 5/2019), and carried out accordingly.

Administration of FN and material sampling for electron and light microscopy. Young and old rats were fed either standard rodent chow (Labofeed H, Wytwornia Pasz Morawski, Kcynia, Poland) (control animals) or the same chow supplemented with $0.1 \%$ FN (Glentham Life Sciences, Corsham, UK) (FN-treated group). For electron microscopy studies, two young and two old animals from each group were anesthetized using ketamine $90 \mathrm{mg} / \mathrm{kg}$ and xylazine $10 \mathrm{mg} / \mathrm{kg}$, i.p. The heart was exposed by slitting the chest and a perfusion needle was inserted into the left ventricle of the heart. At the same time, the continuity of veins falling into the right atrium of the heart was broken. First, a $0.9 \% \mathrm{NaCl}$ solution with 500 units of heparin to prevent blood clotting was injected in order to rinse blood from the circulatory system. Then, a fixative $(2.5 \%$ glutaraldehyde in $0.1 \mathrm{M}$ phosphate buffer ( $\mathrm{pH}$ 7.4)) was administered (approx. $400 \mathrm{ml}$ per $250 \mathrm{~g}$ of animal weight). In the first phase, the fixer was pressed in faster mode $(10 \mathrm{ml} /$ (min), and then the pump flow was halved. After perfusion, the fixative was removed from the pump tubing by rinsing it with saline solution. Liver samples (approx. $1 \mathrm{~mm}^{3}$ ) were collected and immediately placed in the same fixative solution before further processing. For light microscopy examination, three animals per group were sacrificed under full anesthesia (ketamine $90 \mathrm{mg} / \mathrm{kg}$ and xylazine $10 \mathrm{mg} / \mathrm{kg}$, i.p.) through exsanguination from heart puncture, followed by decapitation. Liver samples were collected and immediately frozen in liquid nitrogen for further processing.

Transmission electron microscopy. For ultrastructural examination of hepatocytes, the liver samples of two rats from each group were analyzed. After overnight fixation in $2.5 \%$ glutaraldehyde in $0.1 \mathrm{M}$ phosphate buffer ( $\mathrm{pH} 7.4)$, tissues were treated with $1 \%$ osmium tetroxide in $0.12 \mathrm{M}$ phosphate buffer and embedded in epon resin (Sigma-Aldrich, St. Louis, MO, USA). Ultrathin sections ( $70 \mathrm{~nm}$ ) were cut and, after dehydration, stained with uranyl acetate (Plano GmbH, Wetzlar, Germany) and lead citrate (Electron Microscopy Sciences, Hatfield, PA, USA). Samples were analyzed with a transmission electron microscope (JEOL JEM-1200 EX II, University Park, PA, USA) at an acceleration voltage of $80 \mathrm{kV}$.

Oil Red O staining. Seven- $\mu$ m-thick frozen liver sections of three rats per group were obtained using a cryostat (Leica CM1950, Kawaska, Warsaw, Poland), and mounted on glass slides (SuperFrost Ultra Plus, Thermo Scientific, Vantaa, Finland). The sections were briefly washed in $\mathrm{dH}_{2} \mathrm{O}$, PBS-buffered 4\% paraformaldehyde for $1 \mathrm{~min}$, and briefly in absolute alcohol. Then, the sections were incubated in fresh-diluted $0.5 \%$ Oil Red O solution in isopropyl alcohol (Sigma-Aldrich) for $12 \mathrm{~min}$, briefly washed in $\mathrm{dH}_{2} \mathrm{O}$, and incubated in Harris's hematoxylin solution (Sigma-Aldrich) for $5 \mathrm{~s}$, briefly washed in $\mathrm{dH}_{2} \mathrm{O}$ and $80 \%$ ethanol solution, and embedded in glycerol (Sigma-Aldrich).

Morphometric studies. In the samples of the liver from each group of animals the cytoplasm of randomly selected hepatocytes located in various regions of the ultrathin sections was outlined and its area was measured with the use of the ImageJ software (NIH, Bethesda, MD, USA). Thereafter, the number of mitochondria, peroxisomes, and lipid droplets in chosen area was counted. Basically, the morphometry was performed at the magnification of $10,000 \times$ and $15,000 \times$. The results are shown as the number of the listed structures per unit area of the cytoplasm expressed in square micrometers. 
For the measurement of relative content of lipid droplets in liver samples, two non-contiguous sections from three rats per each group were analyzed, in three to five randomly-selected view fields at the $20 \times$ objective magnification in each section. Microphotographs were obtained using Olympus BX43 microscope equipped with an Olympus UC 90 digital camera (Olympus, Tokyo, Japan).

The relative area occupied in the Oil Red O stained sections by the lipid droplets were analyzed using the cellSens Dimension Software (Olympus, Tokyo, Japan).

Western blotting. The semi-quantification of LC3A/B and beclin 1 proteins in the liver was performed by Western blotting (WB) in 4 samples from every group. Whole-cell lysates were obtained using the Mammalian Cell Extraction Kit (BioVision, Miliptas, CA, USA) and $20 \mu \mathrm{g}$ protein samples were separated on $10 \%$ SDS-PAGE gels, transferred to PVDF membranes (Bio-Rad, Warsaw, Poland), and blocked with $7 \%$ non-fat milk in TBS with $0.1 \%$ Tween 20 (TBST) for $1.5 \mathrm{~h}$. The membranes were incubated overnight at $4^{\circ} \mathrm{C}$ with anti-LC3A/B (Santa Cruz Biotechnology, Dallas, Texas) or anti-beclin 1 (Santa Cruz Biotechnology, Dallas, TX, USA) rabbit polyclonal antibodies at 1:1,000 dilution in 3\% non-fat milk in TBST. Thereafter, the membranes were incubated with HRP-conjugated anti-rabbit secondary antibodies (Sigma-Aldrich) at 1:10,000 dilution in 3\% non-fat milk in TBST for $2 \mathrm{~h}$ at room temperature. Bands were visualized using Clarity Western ECL Substrate (Bio-Rad) and developed using ImageQuant LAS 500 Chemiluminescence CCD Camera (GE Healthcare Life Sciences, Pittsburgh, PA, USA). $\mathrm{LC} 3 \mathrm{~A} / \mathrm{B}$ and beclin1 protein levels were analyzed relative to glyceraldehyde 3-phosphate dehydrogenase (GAPDH) levels (1:50,000 antibody dilution in 3\% non-fat milk in TBST; Sigma-Aldrich) using QuantityOne Software (Bio-Rad).

Statistical analysis. Statistical analyses were performed using GraphPad Prism v. 3.0 software (GraphPad Inc., San Diego, CA, USA). After applying Shapiro-Wilk test for normality and Grubbs test for outliers, statistical analyses were performed using Student $t$-test (for relative area density of organelles and relative area of lipid droplets) or nonparametric Kruskal-Wallis ANOVA (for proteins' expression in WB). Data are presented as mean \pm SD. Statistical significance was set at $p<0.05$.

\section{Results}

\section{Ultrastructure of hepatocytes of young and old rats}

In control young rats, electron microscopy revealed well-preserved ultrastructure of hepatocytes. The cells presented normal structure of numerous mitochondria, abundant rough endoplasmic reticulum (RER), and numerous peroxisomes and lysosomes (Figs. 1B and 2B; Suppl. Figs. 2 and 4), glycogen stored in a form of 'rosettes' (Figs. 1B and 2A; Suppl. Fig. 4) and lipid droplets (Suppl. Figs. 1 and 3). Some hepatocytes in the liver of young rats were binucleated (Fig. 1A; Suppl. Fig. 2).

In control old rats, electron microscopy revealed, similarly to young ones, well-preserved ultrastructure of hepatocytes (Figs. 1C, 1D, 2C, and 2D). However, in comparison to young animals, hepatocytes of old control animals contained relatively more electron-dense mitochondria (Figs. 1C, 1D, 2C, and 2D), well-developed RER (Figs. 1D, 2C; Suppl. Figs. 5 and 6), and some autolysosomes (Figs. 1C and 2D; Suppl. Figs. 6 and 8). Moreover, hepatocytes of control old rats appeared to have more lipid droplets (Fig. 1D), peroxisomes (Fig. 2D; Suppl. Fig. 8) and glycogen 'rosettes' (Figs. 2C and 2D; Suppl. Figs. 6 and 7) than hepatocytes of young animals. Binucleated and polyploid hepatocytes seemed to occur more often in the liver of old rats (Fig. 1C).

In comparison to young control animals, hepatocytes of FN-treated young rats revealed numerous mitochondria with more electron-dense matrix (Fig. 3A; Suppl. Figs. 9 and 10), more peroxisomes and lysosomes (Figs. 3A, 3B, and 4B; Suppl. Figs. 9 and 10) and occasionally autophagosomes (Suppl. Fig. 9) and autophagic vacuoles (Fig. 4A). Some lipid droplets were also seen (Fig. 4A). Interestingly, the intercellular spaces were distended (Fig. 3A).

In comparison to old control animals, hepatocytes of FN-treated old rats appeared to have more mitochondria, peroxisomes, lysosomes, RER cisternae (Figs. 3C and 3D; Suppl. Figs. 11) and glycogen 'rosettes' (Figs. 4C and 4D). Compared with the hepatocytes of control rats, the number of lipid droplets seemed to be lower in the cells of FN-treated old animals (Fig. 3C; Suppl. Fig. 11) whereas the content of autophagosomes and autolysosomes seemed to be more variable (Figs. 4C and 4D).

In the livers of both control and FN-treated young and old rats, hepatocytes were connected by numerous junctional complexes with prominent desmosomes (Figs. 1D and 3C; Suppl. Figs. 5 and 11).

\section{Morphometric analysis at the electron microscopic level}

Because the qualitative analysis of electron-microscopic photographs suggested the presence of some age- or FN-related changes in the number of hepatocytes' organelles, we performed semi-quantitative morphometric analysis to determine relative area density of mitochondria, peroxisomes, and lipid droplets in hepatocytes of each experimental group.

We found that in the hepatocytes of old control rats the relative number of mitochondria and peroxisomes was lower by $29 \%(p<0.05)$ and $57 \%$ $(p<0.05)$, respectively, than in hepatocytes of con- 

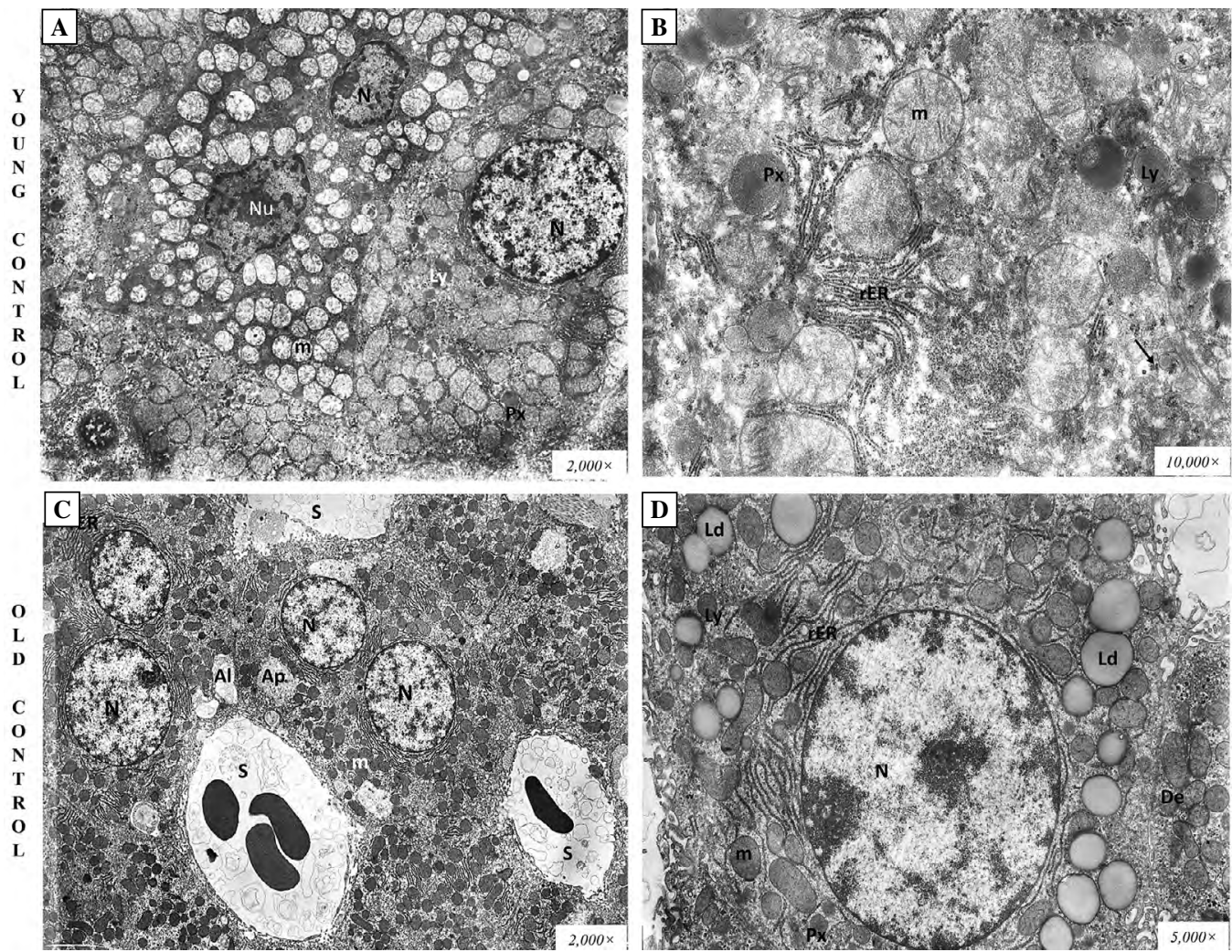

Figure 1. EM micrographs of hepatocytes of control young and old rats at lower magnifications. Abbreviations: Al — autolysosome; Ap — autophagosome; col — collagen fibers; De — desmosome; Ld — lipid droplet; Ly — lysosome; $\mathrm{m}$ - mitochondrion; N — nucleus; Px — peroxisome; rER — rough endoplasmic reticulum; S — sinusoid; arrow, glycogen rosette.
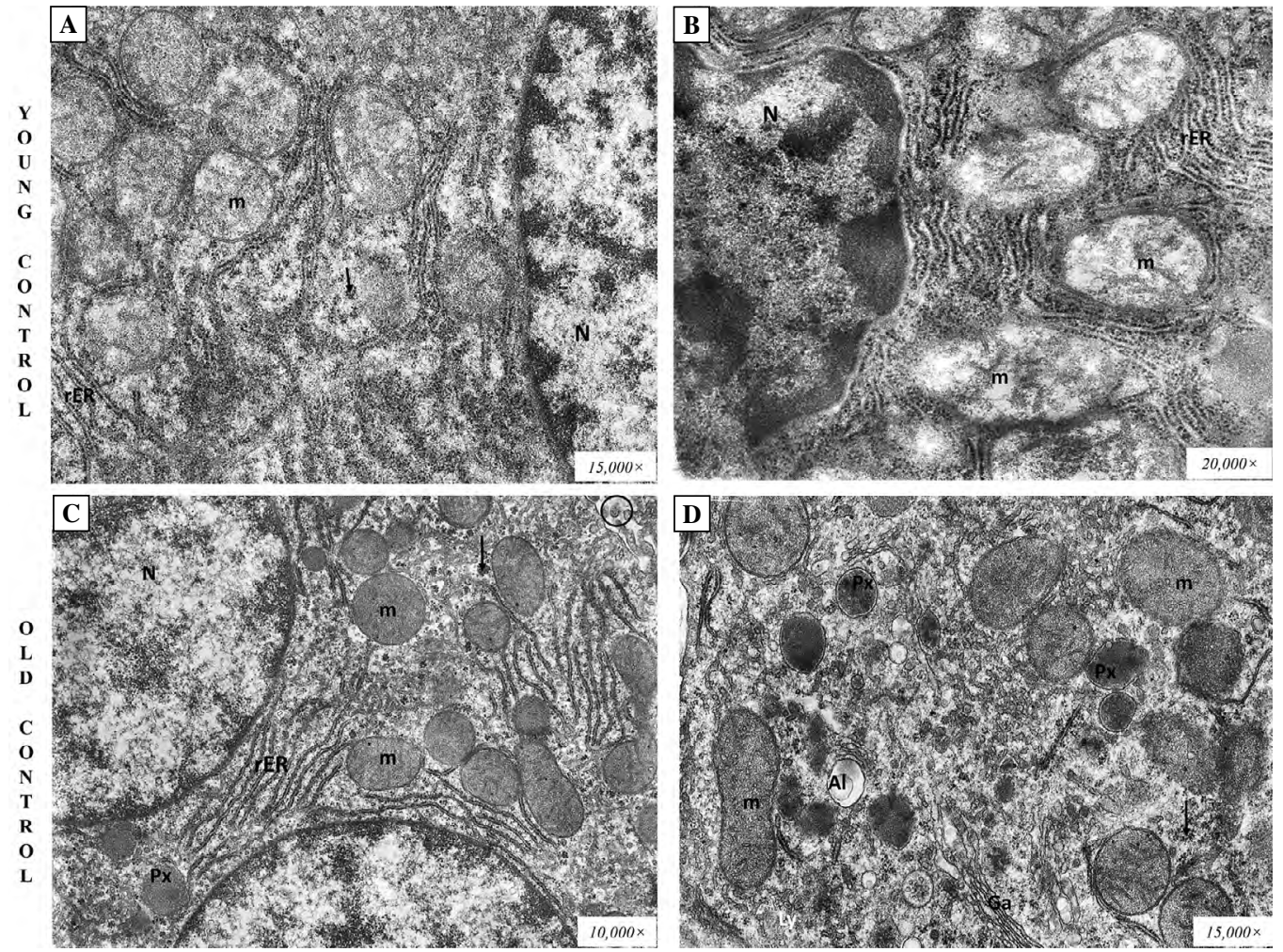

Figure 2. EM micrographs of hepatocytes of control young and old rats at higher magnifications. Abbreviations as in the description of Figure 1 and: $\mathrm{Ga}$ - Golgi apparatus, black circle — bile canaliculus. 

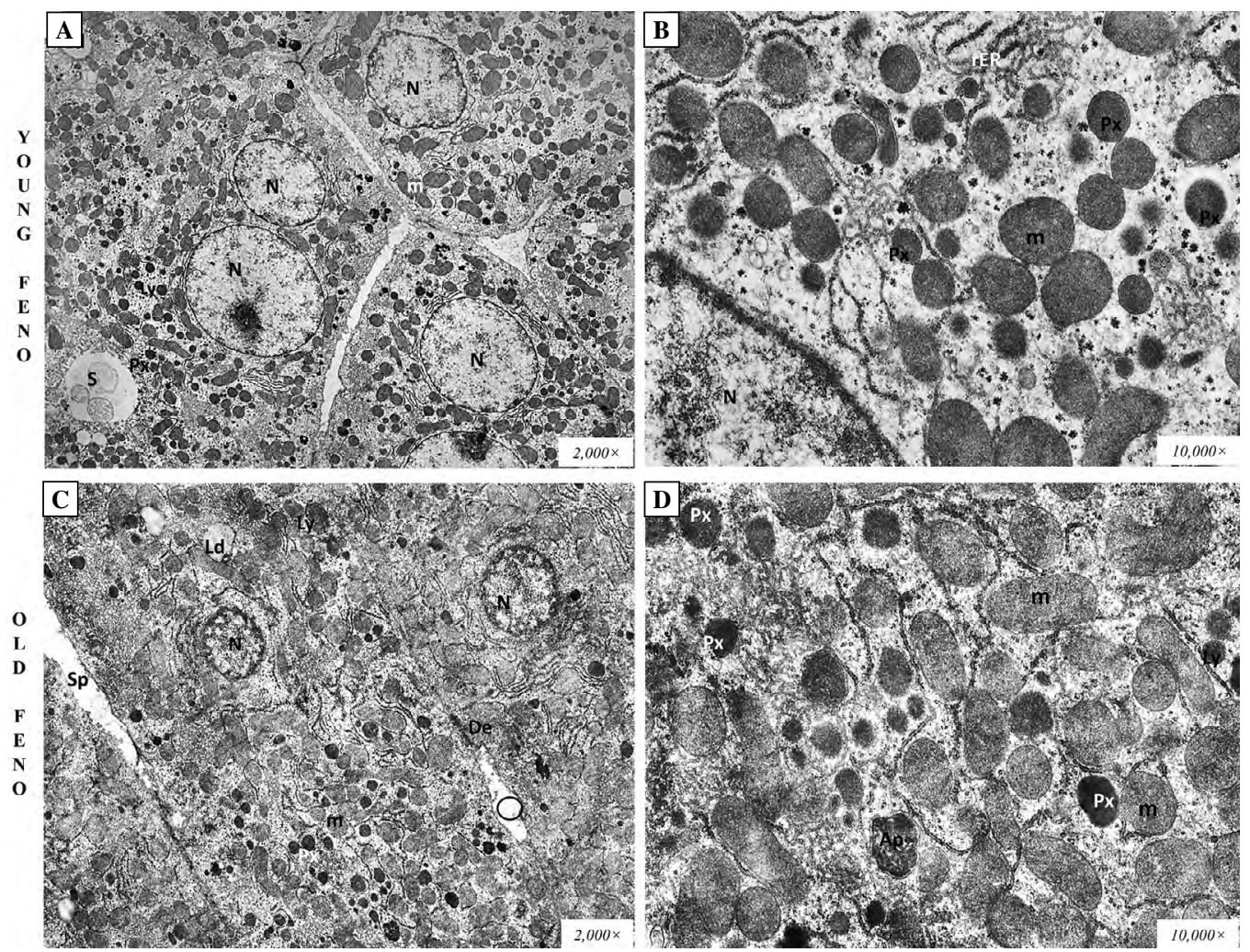

Figure 3. EM micrographs of hepatocytes of FN-treated (feno) young and old rats at lower magnifications. Abbreviations as in the description of Figure $1 \& 2$ : Ap - autophagosome; Sp - lateral recess of the space of Disse.
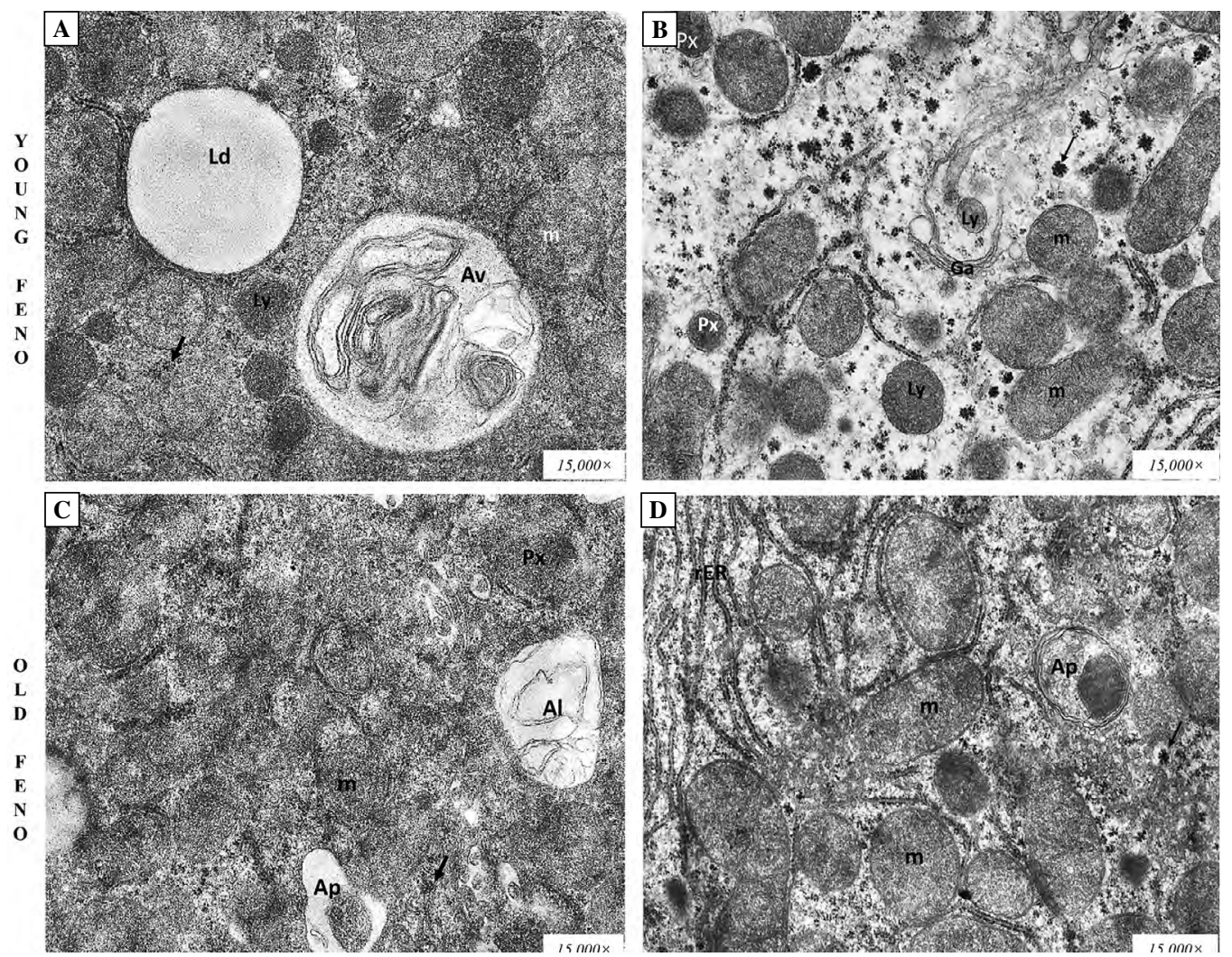

Figure 4. EM micrographs of hepatocytes of FN-treated (feno) young and old rats at higher magnification. Abbreviations as in the description of Figures 1, 2 and 3. 


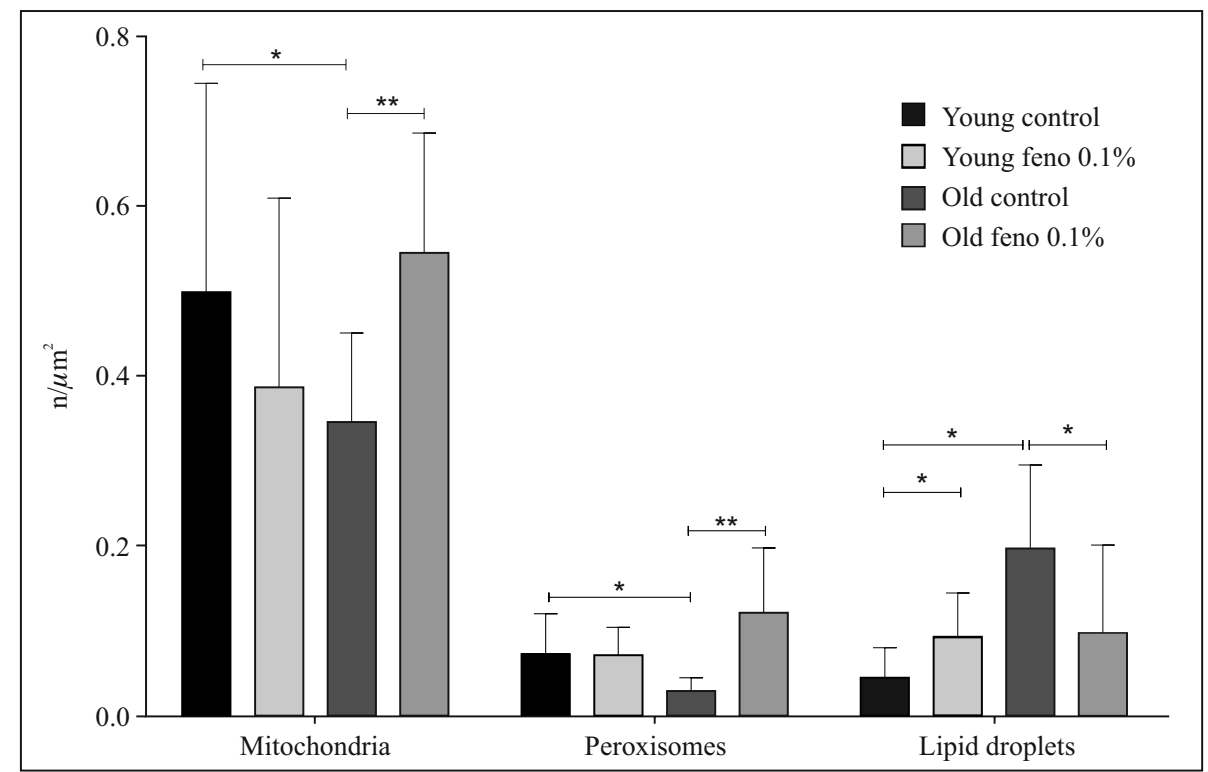

Figure 5. Relative area density of mitochondria, peroxisomes, and lipid droplets in hepatocytes of young and old control and FN-treated (feno) rats measured at the ultrastructural level and expressed as number of organelles per $\mu \mathrm{m}^{2}$. Data are presented as mean $\pm \mathrm{SD}, \mathrm{n}=2$ per group, ${ }^{*} \mathrm{p}<0.05, * * \mathrm{p}<0.01$ (Student's $t$-test).

trol young rats. FN treatment increased the number of mitochondria and peroxisomes in hepatocytes of old rats by $57 \%$ and $300 \%$, respectively $(p<0.05$ and $p<0.01$ ). However, FN did not affect the number of mitochondria or peroxisomes in hepatocytes of young rats (Fig. 5).

We also found that in hepatocytes of control old rats the relative number of lipid droplets was 3 -fold higher than in cells of young animals $(p<0.01)$ (Fig. 5). FN treatment decreased the number of lipid droplets by $50 \%(p<0.05)$ in hepatocytes of old rats, and increased it by $50 \%$ in cells of young animals (Fig. 5).

\section{Relative content of lipid droplets measured in liver sections stained by Oil Red $O$}

The heterogeneity of the liver lobule has been described for some basic metabolic pathways $[15,16]$. Since our EM study had basically qualitative character and the sampled pieces of liver tissue were derived from the middle part of classic hepatic lobules (zone 2 of portal lobule), we decided to test the expression of lipid droplets across the liver lobule in cryostat sections stained with Oil Red O. This method made it possible to asses semi-quantitatively the relative area occupied by lipid droplets in the liver sections. In the livers of control both young and old rats, Oil Red O staining revealed the presence of lipid droplets mainly in the hepatocytes of the periportal zone of the classic hepatic lobule (zone 1 of portal lobule); however, the intensity of the staining reaction was much higher in the liver of old rats (Fig. 6).
The FN treatment resulted in more homogenous distribution of lipid droplets within hepatic lobules and seemingly lower staining intensity (Fig. 6B, D) which prompted us to assess drug's action by a morphometric method. It revealed that the relative area density of lipid droplets was higher in the liver of control old rats by $371 \%(p<0.01)$ than in young ones. The treatment of both young and old rats with $0.1 \% \mathrm{FN}$ did not cause any significant changes in the area density of lipid droplets in young and old rats $(p>0.05)$ (Fig. 6E).

\section{Expression of pro-autophagic proteins $L C 3 A / B$ and beclin 1}

One of the features of cell senescence is decreased number of autophagic structures/vacuoles [17]. Western blot method was used to assess the relative levels of $\mathrm{LC} 3 \mathrm{~A} / \mathrm{B}$ and beclin 1 proteins in the homogenates of rat livers. We did not observe any age-related changes, although there was a notable tendency towards lower expression of LC3A/B and increased expression of beclin 1 in control old rats as compared to young ones. The FN treatment was not associated with any significant changes in the expression levels of autophagy-related proteins LC3A/B and beclin 1 in the liver of either young and old rats (Fig. 7).

\section{Discussion}

Fenofibrate has been approved for treating dyslipidemias; however, its effects on the liver ultrastructure in 


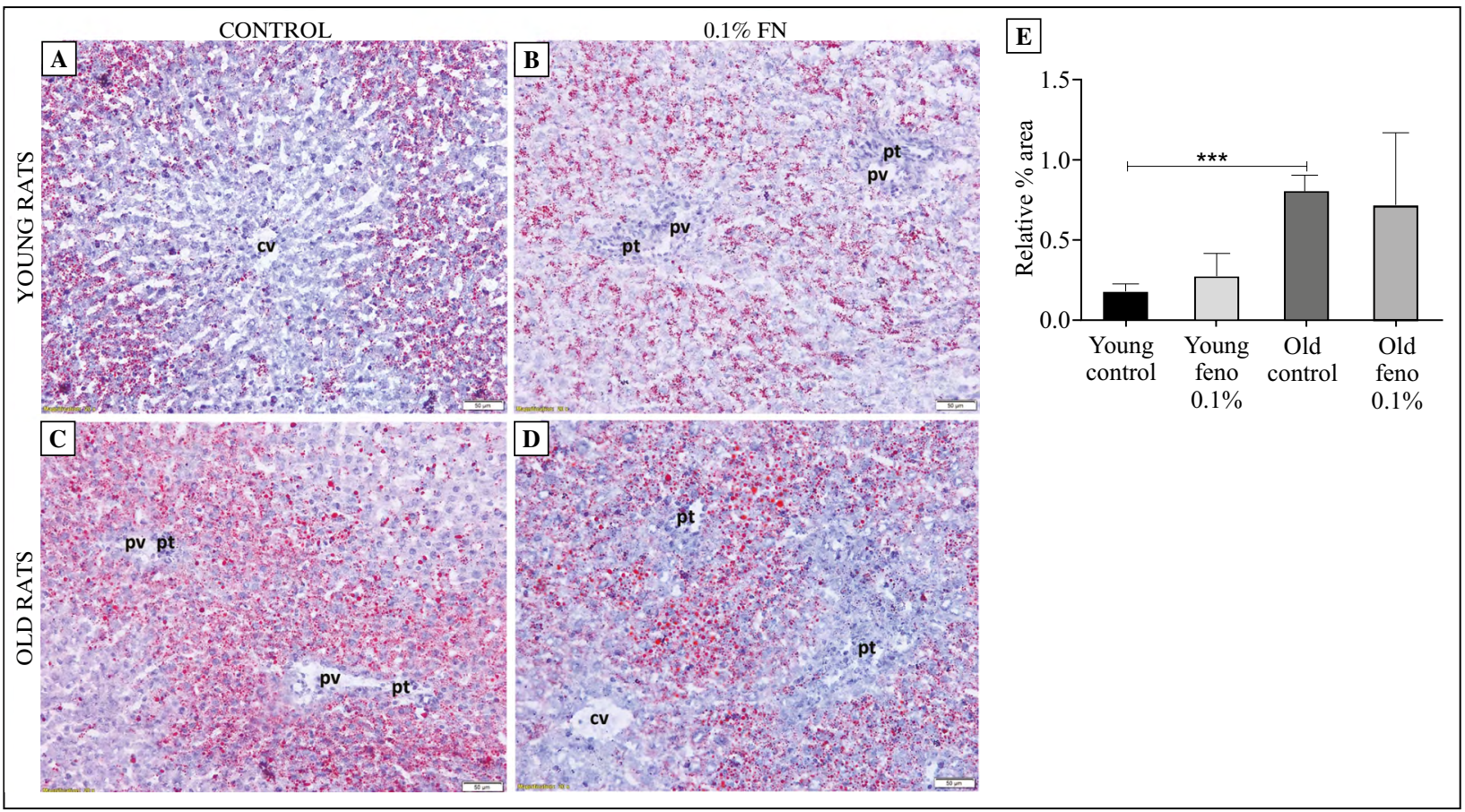

Figure 6. Oil Red O staining of the liver of control and FN-treated (feno) young and old rats. The most representative microphotographs at $200 \times$ magnification are shown (A-D). Relative area of lipid droplets (E) was assessed; data are presented as mean $\pm \mathrm{SD}$ of 3 animals per group, ${ }^{* *} \mathrm{p}<0.01$ (Student $t$-test).

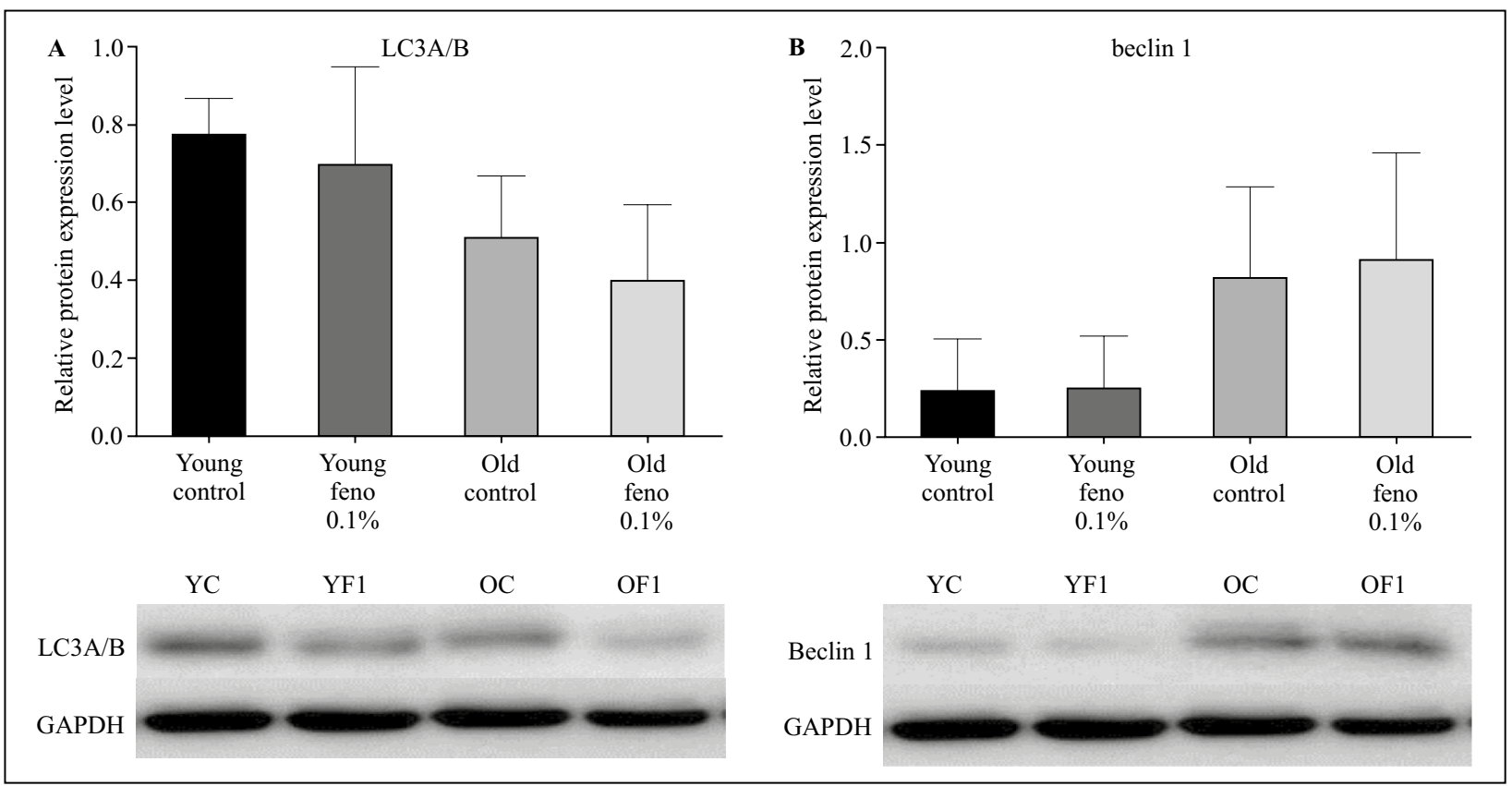

Figure 7. Protein expression of LC3A/B and beclin 1 in the liver of control and FN-treated (feno) young and old rats. Relative protein levels were determined by Western blotting. Representative blots are shown. Data are presented as mean \pm SD, 4 animals per group. No significant changes were found $(\mathrm{p}>0.05)$. Abbreviations: YC - control young rats; YF1 - young rats treated with $0.1 \% \mathrm{FN}$; OC — control old rats; OF1 — old rats treated with $0.1 \%$ FN (Kruskal-Wallis ANOVA).

the context of aging have not been thoroughly investigated. Our study revealed some important differences in the submicroscopic structure of hepatocytes of young and old male rats which were subjected to shortterm, 30-day-long FN treatment. We suggest that it may reflect changes in liver function, as indicated by 
changes in biochemical serum parameters and liver morphology described in our previous report [8].

It has been showed that aging in rodents is associated with some decline of mitochondrial function with mitochondria from old mice characterized by less regular and more heterogeneous cristae not arranged in parallel stacks [18], and increased content of oxidation products of phospholipids, proteins and DNA, decreased membrane potential, and increased mitochondrial size and fragility [19]. It was revealed that aging in rodents [20-22] and humans [22] is associated with the increase in individual mitochondrial volume and decline in the number of mitochondria in the liver. These findings are supported by our observation of the increased matrix electron density of mitochondria in hepatocytes of old rats. Our finding of the lower relative number of mitochondria in hepatocytes of control old rats as compared to young ones was reported by some, however not all, investigators. E.g. much higher decline (3.5 times) was found in the relative number of mitochondrial profiles in the livers of 24-month-old control mice as compared with 6-month old animals [23]. In hepatocytes isolated from 22-23 and 32-36 month-old Wistar rats the number of mitochondria per cell was similar; however, mitochondria of old animals were larger than mitochondria in hepatocytes of 3-4 month-old rats that was explained by age-related increase in mitochondrial matrix volume [24]. Electron cryotomography showed that in the mitochondria isolated from the livers of old (80-96-weekold) mice mitochondrial cristae were less regular and not arranged in parallel stacks, less interconnected, generally wider and not spanning the entire mitochondrion in comparison with mitochondria from livers of 20-week-old animals [18]. Łysek-Gładysińska et al. observed increased number of damaged mitochondria in the hepatocytes of 17-month-old mice as compared to 6-month old animals [21]. Interestingly, no age-dependent increase in hydrogen peroxide release relative to the amount of oxygen consumed was observed in the isolated murine liver mitochondria [18].

The short-term FN treatment of old rats resulted in the appearance more electron-dense mitochondria in hepatocytes of both young and old animals than in control ones. Moreover, FN increased relative number of mitochondria only in hepatocytes of old rats that was lower in control old animals as compared to young ones. Since mitochondria play a major role in the energy homeostasis, this novel observation may indicate beneficial effect of FN that upregulated age-related decline of the number of mitochondria in rat hepatocytes.

Peroxisomes, abundant in cells that are involved in lipid and xenobiotics metabolism, play a major role in the breakdown of long-chain fatty acids, thereby complementing mitochondrial fatty-acid oxidation [25]. In our study we demonstrated that the age is associated with the decrease in relative number of peroxisomes in hepatocytes, and $\mathrm{FN}$ administration increases the relative number of peroxisomes only in hepatocytes of old rats. Together with the effect of FN on the relative number of mitochondria in hepatocytes of old rats this finding suggests beneficial effects of FN on the on the hepatic lipid and energy metabolism in aged rodents. Although FN was shown to increase the number of peroxisomes in the liver of juvenile (5-week-old) male Wistar rats after two weeks of $0.1 \% \mathrm{FN}$ administration [26], this effect was not observed by us in the liver of adult, sexually mature animals. The discrepancy between our data and those of the other authors could be attributed to differences between rat strains [26, 27]. For example, in the liver of 27-month-old male Fischer 344 rats, clofibric acid, one of the model fibrates, induced only slight proliferation of peroxisomes [28]. Moreover, FN did not cause proliferation of peroxisomes in the liver of rhesus monkeys (treatment with $200 \mathrm{mg} / \mathrm{kg}$ body weight/day FN for 12 months) [29]. It is also possible that the chosen dose of FN or the duration of its administration were too short to induce proliferation of peroxisomes in hepatocytes of young and old rat.

It is widely known that aging is associated with lipid accumulation in the liver [30]. In our study we reveal that old age of rats was associated with the increase in relative number of lipid droplets within single hepatocytes and in liver sections at the light microscopy level. Similar increase in lipid droplets were found in the liver of aging mice [21,31]. Interestingly, in Fischer 344 male rats the volume of lipid droplets in hepatocytes undergoes a biphasic response during aging, i.e. gradual post-maturational increase is followed by the decline during late senescence $[22,32]$. The beneficial effect of FN on the relative number of lipid droplets in hepatocytes of old rats was not observed in young animals in whom only a slight increase was found. This discrepancy may be caused by originally higher content of lipid droplets in old hepatocytes and, probably, increased sensitivity of old rats' hepatocytes to the action of FN, as observed also for the relative number of mitochondria and peroxisomes. In contrast to these ultrastructural observations, FN did not alter the relative area occupied by lipid droplets detected by Oil Red O staining at the light microscopy level. This may be due to the accumulation of lipid vacuoles in hepatic stellate cells which were not analyzed by electron-microscopy morphometry. We are not aware of any studies that investigated effects of FN on the liver fat-storing (or Ito) cells. Similar effect was observed 
in hyperlipidemic patients with the presence of lipid droplets in the liver cells and numerous hepatocytes containing medium- or large-size lipid droplets in 6 out of $28 \mathrm{FN}$-treated patients [33].

Autophagosomes and autolysosomes play a critical role in maintaining normal intracellular homoeostasis in response to various stresses, and autophagy, the process associated with the formation of this structures, controls the quality and quantity of cellular organelles and macromolecules [34]. The liver presents high levels of metabolic-stress-induced autophagy that is under the regulation of hormones and amino acids' concentrations. Liver autophagy provides starved cells with amino acids, glucose and free fatty acids for use in energy production and synthesis of new macromolecules. It is also known that liver autophagy contributes to basic hepatic functions, including glycogenolysis, gluconeogenesis and $\beta$-oxidation, through selective turnover of specific cargos controlled by a series of transcription factors, e.g. CREB (cAMP response element) or PPAR $\alpha$ [17, 34]. Impaired or deficient autophagy is believed to be associated with aging, and several age-related pathologies [17].

In our study, ultrastructural examination of hepatocytes revealed the presence of autophagosomes and autolysosomes in both age groups. It was showed that in C57BL/6 female mice the number of autophagic structures/vacuoles in hepatocytes decreased with age, and suggested that the age-related decrease in autophagy in the liver may induce accumulation of cellular materials in the liver of aged mice [17]. The study on 26-month-old male Fisher rats showed that there was no effect of age on the fractional volume of autophagic vacuoles [35]. In our study we did not found age-related differences in hepatic expression of LC3A/B (related to autophagosome formation) and beclin 1 proteins (a common regulator of autophagy and apoptosis). Other authors reported that in old compared to young rats, expression of beclin 1, LC3-1 and procathepsin $\mathrm{D}$ was decreased, and expression of cathepsin $\mathrm{D}$ (a product of procathepsin $\mathrm{D}$ proteolytic activation in lysosomes) was increased [35].

Our examination of hepatocytes ultrastructure revealed the presence of autophagosomes and autolysosomes in young and old FN-treated rats. Recent studies showed that hepatic autophagy is activated via $\operatorname{PPAR} \alpha$ after short-term treatment with $\operatorname{PPAR} \alpha$ agonists both in vivo and in vitro [36, 37]. There are some data suggesting that treatment with FN may have various effects on autophagy in hepatocytes. In neonate, 5-day-old G6pc ${ }^{-/}$mice (autosomal mutation in glucose-6-phosphatase; a model of neonatal glycogen storage disease type Ia) 5-day-long treatment with FN induced autophagy in the liver accompanied by increased hepatic LC3-II and beclin 1 protein expression, and TEM analyses revealed numerous autophagosomes with electron-dense glycogen granules [14]. In hepatocytes isolated from 8-week-old male C57BL/6J mice treated with FN $(0.5-5 \mu \mathrm{g} / \mathrm{ml}$ for 24 hours), the drug exerted a protective effect against acetaminophen (APAP)-induced hepatotoxicity by enhancing autophagy via elevation of LC3-II and degradation of p62 proteins [23]. In contrast, Zhu et al. observed no effect of FN on the process of autophagy in monosodium glutamate (MSG)-induced obese mice that were treated with $\mathrm{FN}$ for 5 weeks at dose $45 \mathrm{mg} / \mathrm{kg}$ [38].

In summary, the obtained results suggest that fenofibrate may have beneficial effects on the energy metabolism of old rats by increasing the number of mitochondria and peroxisomes in hepatocytes.

\section{Acknowledgments}

The authors are grateful to Drs. M. Szaryńska and A. Olejniczak-Kęder for kind supply of anti-LC1/II and anti-beclin 1 antibodies.

\section{Funding}

The research was financed by the statutory grant (ST12) of the Medical University of Gdansk, Gdansk, Poland.

\section{Declaration of competing interest}

The authors report no declarations of interest.

\section{Authors' contribution}

Adrian Zubrzycki: conceptualization, methodology, formal analysis, investigation, resources, writing original draft, visualization, project administration. Agata Wronska: conceptualization, methodology, formal analysis, investigation, resources, writing original draft, visualization, project administration. Agata Zauszkiewicz-Pawlak: methodology, investigation, resources. Zbigniew Kmiec: conceptualization, formal analysis, resources, writing - original draft, writing - review \& editing, supervision, project administration, funding acquisition.

\section{References}

1. Mancini G, Hegele R, Leiter L. Dyslipidemia. Can J Diabetes. 2018; 42: S178-S185, doi: 10.1016/j.jcjd.2017.10.019, indexed in Pubmed: 29650093.

2. Sahebkar A, Simental-Mendía LE, Watts GF, et al. Lipid and Blood Pressure Meta-analysis Collaboration (LBPMC) Group. Comparison of the effects of fibrates versus statins on 
plasma lipoprotein(a) concentrations: a systematic review and meta-analysis of head-to-head randomized controlled trials. BMC Med. 2017; 15(1): 22, doi: 10.1186/s12916-017-0787-7, indexed in Pubmed: 28153024.

3. Sairyo M, Kobayashi T, Masuda D, et al. A novel selective PPAR $\alpha$ modulator (SPPARM $\alpha$ ), K-877 (Pemafibrate), attenuates postprandial hypertriglyceridemia in mice. J Atheroscler Thromb. 2018; 25(10): 1086, doi: 10.5551/jat.ER39693, indexed in Pubmed: 30197400.

4. Bougarne N, Weyers B, Desmet SJ, et al. Molecular actions of PPAR $\alpha$ in lipid metabolism and inflammation. Endocr Rev. 2018; 39(5): 760-802, doi: 10.1210/er.2018-00064, indexed in Pubmed: 30020428.

5. Pawlak M, Lefebvre P, Staels B. Molecular mechanism of PPAR $\alpha$ action and its impact on lipid metabolism, inflammation and fibrosis in non-alcoholic fatty liver disease. J Hepatol. 2015; 62(3): 720-733, doi: 10.1016/j.jhep.2014.10.039, indexed in Pubmed: 25450203.

6. Livertox. LiverTox: Clinical and research information on drug-induced liver injury. Fenofibrate. 2015. Available at: https://www.ncbi.nlm.nih.gov/books/NBK548607/. Accessed August.; 5: 2021.

7. Pettersen JC, Pruimboom-Brees I, Francone OL, et al. The PPAR $\alpha$ agonists fenofibrate and CP-778875 cause increased $\beta$-oxidation, leading to oxidative injury in skeletal and cardiac muscle in the rat. Toxicol Pathol. 2012; 40(3): 435-447, doi: 10.1177/0192623311431945, indexed in Pubmed: 22301950.

8. Zubrzycki A, Wrońska A, Kotulak-Chrząszcz A, et al. Fenofibrate impairs liver function and structure more pronounced in old than young rats. Arch Gerontol Geriatr. 2020 [Epub ahead of print]; 91: 104244, doi: 10.1016/j.archger.2020.104244, indexed in Pubmed: 32927318.

9. Cindoruk M, Kerem M, Karakan T, et al. Peroxisome proliferators-activated alpha agonist treatment ameliorates hepatic damage in rats with obstructive jaundice: an experimental study. BMC Gastroenterol. 2007; 7: 44, doi: 10.1186/1471230X-7-44, indexed in Pubmed: 18045488.

10. Colton HM, Falls JG, Ni H, et al. Visualization and quantitation of peroxisomes using fluorescent nanocrystals: treatment of rats and monkeys with fibrates and detection in the liver. Toxicol Sci. 2004; 80(1): 183-192, doi: 10.1093/toxsci/kfh144, indexed in Pubmed: 15084755.

11. Steinberg P, Schladt L, Dienes HP, et al. Microsomal and cytosolic epoxide hydrolases, the peroxisomal fatty acid beta-oxidation system and catalase. Activities, distribution and induction in rat liver parenchymal and non-parenchymal cells. Eur J Biochem. 1988; 176(1): 39-45, doi: 10.1111/j.14321033.1988.tb14248.x, indexed in Pubmed: 3416872.

12. Yang Q, Nagano T, Shah Y, et al. The PPAR alpha-humanized mouse: a model to investigate species differences in liver toxicity mediated by PPAR alpha. Toxicol Sci. 2008; 101(1): 132-139, doi: 10.1093/toxsci/kfm206, indexed in $\mathrm{Pu}-$ bmed: 17690133.

13. de la Rosa Rodriguez MA, Sugahara Go, Hooiveld GJ, et al. The whole transcriptome effects of the PPAR $\alpha$ agonist fenofibrate on livers of hepatocyte humanized mice. BMC Genomics. 2018; 19(1): 443, doi: 10.1186/s12864-018-4834-3, indexed in Pubmed: 29879903.

14. Yavarow ZA, Kang HR, Waskowicz LR, et al. Fenofibrate rapidly decreases hepatic lipid and glycogen storage in neonatal mice with glycogen storage disease type Ia. Hum Mol Genet. 2020; 29(2): 286-294, doi: 10.1093/hmg/ddz290, indexed in Pubmed: 31816064.

15. Halpern KB, Shenhav R, Matcovitch-Natan O, et al. Single-cell spatial reconstruction reveals global division of labour in the mammalian liver. Nature. 2017; 542(7641): 352-356, doi: 10.1038/nature21065, indexed in Pubmed: 28166538.

16. Ben-Moshe S, Itzkovitz S. Spatial heterogeneity in the mammalian liver. Nat Rev Gastroenterol Hepatol. 2019; 16(7): 395-410, doi: 10.1038/s41575-019-0134-x, indexed in Pubmed: 30936469.

17. Uddin MN, Nishio N, Ito $\mathrm{S}$, et al. Autophagic activity in thymus and liver during aging. Age (Dordr). 2012; 34(1): 75-85, doi: 10.1007/s11357-011-9221-9, indexed in Pubmed: 21387084.

18. Brandt T, Mourier A, Tain LS, et al. Changes of mitochondrial ultrastructure and function during ageing in mice and Drosophila. Elife. 2017; 6, doi: 10.7554/eLife.24662, indexed in Pubmed: 28699890.

19. Shigenaga MK, Hagen TM, Ames BN. Oxidative damage and mitochondrial decay in aging. Proc Natl Acad Sci U S A. 1994; 91(23): 10771-10778, doi: 10.1073/pnas.91.23.10771, indexed in Pubmed: 7971961.

20. de la Cruz J, Burón I, Roncero I. Morphological and functional studies during aging at mitochondrial level. Action of drugs. Int J Biochem. 1990; 22(7): 729-735, doi: 10.1016/0020-711x(90)90008-q, indexed in Pubmed: 2401374.

21. Łysek-Gładysińska M, Wieczorek A, Jóźwik A, et al. Aging-related changes in the ultrastructure of hepatocytes and cardiomyocytes of elderly mice are enhanced in ApoE-deficient animals. Cells. 2021; 10(3), doi: 10.3390/cells10030502, indexed in Pubmed: 33652838.

22. Schmucker DL. Aging and the liver: An Update. J Gerontol A Biol Sci Med Sci. 1998; 53A(5): B315-B321, doi: 10.1093/ gerona/53a.5.b315.

23. Nagata T. Electron microscopic radioautographic study on protein synthesis in hepatocyte mitochondria of aging mice. Scientific World Journal. 2006; 6: 1583-1598, doi: 10.1100/ tsw.2006.265, indexed in Pubmed: 17173177.

24. Le Couteur DG, Warren A, Cogger VC, et al. Old age and the hepatic sinusoid. Anat Rec (Hoboken). 2008; 291(6): 672-683, doi: 10.1002/ar.20661, indexed in Pubmed: 18484614.

25. He A, Dean JM, Lodhi IJ. Peroxisomes as cellular adaptors to metabolic and environmental stress. Trends Cell Biol. 2021; 31(8): 656-670, doi: 10.1016/j.tcb.2021.02.005, indexed in Pubmed: 33674166.

26. van Zutphen T, Ciapaite J, Bloks VW, et al. Malnutrition-associated liver steatosis and ATP depletion is caused by peroxisomal and mitochondrial dysfunction. J Hepatol. 2016; 65(6): 1198-1208, doi: 10.1016/j.jhep.2016.05.046, indexed in Pubmed: 27312946.

27. Mitchell AM, Lhuguenot JC, Bridges JW, et al. Identification of the proximate peroxisome proliferator(s) derived from di(2-ethylhexyl) phthalate. Toxicol Appl Pharmacol. 1985; 80(1): 23-32, doi: 10.1016/0041-008x(85)90097-3, indexed in Pubmed: 4024105.

28. Yamoto T, Ohashi Y, Teranishi M, et al. Age-related changes in the susceptibility to clofibric acid, a hypolipidemic agent, of male rat liver. Toxicol Lett. 1995; 78(2): 141-145, doi: 10.1016/0378-4274(94)03247-5, indexed in Pubmed: 7618179.

29. Blanc EF, Pinaroli F. Fenofibrate: animal toxicology in relation to side-effects in man (author's transl). Nouv Presse Med 1980; 9: 3737-3746. , indexed in Pubmed: 7208340.

30. Johnson AA, Stolzing A. The role of lipid metabolism in aging, lifespan regulation, and age-related disease. Aging Cell. 2019; 18(6): e13048, doi: 10.1111/acel.13048, indexed in Pubmed: 31560163 .

31. Wan J, Wu X, Chen H, et al. Aging-induced aberrant RAGE/ $\operatorname{PPAR} \alpha$ axis promotes hepatic steatosis via dysfunctional mitochondrial $\beta$ oxidation. Aging Cell. 2020; 19(10): e13238, doi: 10.1111/acel.13238, indexed in Pubmed: 32936538. 
32. Schmucker DL, Mooney JS, Jones AL. Stereological analysis of hepatic fine structure in the Fischer 344 rat. Influence of sublobular location and animal age. J Cell Biol. 1978; 78(2): 319-337, doi: 10.1083/jcb.78.2.319, indexed in Pubmed: 690169.

33. Blümcke S, Schwartzkopff W, Lobeck H, et al. Influence of fenofibrate on cellular and subcellular liver structure in hyperlipidemic patients. Atherosclerosis. 1983; 46(1): 105-116, doi: 10.1016/0021-9150(83)90169-7, indexed in Pubmed: 6838687.

34. Ueno T, Komatsu M. Autophagy in the liver: functions in health and disease. Nat Rev Gastroenterol Hepatol. 2017; 14(3): 170-184, doi: 10.1038/nrgastro.2016.185, indexed in Pubmed: 28053338.

35. Wohlgemuth SE, Julian D, Akin DE, et al. Autophagy in the heart and liver during normal aging and calorie restric- tion. Rejuvenation Res. 2007; 10(3): 281-292, doi: 10.1089/ rej.2006.0535, indexed in Pubmed: 17665967.

36. Jo E, Li S, Liang Q, et al. Chronic activation of PPAR $\alpha$ with fenofibrate reduces autophagic proteins in the liver of mice independent of FGF21. PLoS One. 2017; 12(4): e0173676, doi: 10.1371/journal.pone.0173676, indexed in Pubmed: 28422956.

37. Park JSu, Kang DH, Lee DaH, et al. Fenofibrate activates Nrf2 through p62-dependent Keap1 degradation. Biochem Biophys Res Commun. 2015; 465(3): 542-547, doi: 10.1016/j. bbrc.2015.08.056, indexed in Pubmed: 26282199.

38. Zhu S, Wu Y, Ye X, et al. FGF21 ameliorates nonalcoholic fatty liver disease by inducing autophagy. Mol Cell Biochem. 2016; 420(1-2): 107-119, doi: 10.1007/s11010-016-2774-2, indexed in Pubmed: 27435856.

Submitted: 5 August, 2021 Accepted after reviews: 24 August, 2021 Available as AoP: 13 September, 2021 